\title{
On boundary behavior of Cauchy integrals
}

\begin{abstract}
In this paper, we shall estimate the growth order of the $n$-th derivative Cauchy integrals at a point in terms of the distance between the point and the boundary of the domain. By using the estimate, we shall generalize Plemelj-Sokthoski theorem. We also consider the boundary behavior of generalized Cauchy integrals on compact bordered Riemann surfaces.
\end{abstract}

1. Introduction. Let $\varphi$ be a continuous function on a smooth Jordan curve $\Gamma$ in $\mathbb{C}$ and consider the Cauchy integral;

$$
F(z)=\frac{1}{2 \pi i} \int_{\Gamma} \frac{\varphi(\zeta)}{\zeta-z} d \zeta
$$

for $z \in \mathbb{C} \backslash \Gamma$. It is a holomorphic function on $\mathbb{C} \backslash \Gamma$. Let $D_{+}$and $D_{-}$ denote the bounded component of $\mathbb{C} \backslash \Gamma$ and the unbounded component, respectively. On the boundary behavior of the Cauchy integral, the following is well known and it is called Plemelj-Sokthotski formula (cf. [6]).

Theorem (Plemelj-Sokthotski). Suppose that $\varphi$ is a Hölder continuous function of order $\alpha(0<\alpha<1)$ on $\Gamma$, that is, there exists a constant $A>0$ such that

$$
\left|\varphi\left(\zeta_{1}\right)-\varphi\left(\zeta_{2}\right)\right| \leq A\left|\zeta_{1}-\zeta_{2}\right|^{\alpha}
$$

2010 Mathematics Subject Classification. Primary 30F40; Secondary 30C35.

Key words and phrases. Cauchy integral, Plemelj-Sokthoski theorem, Riemann surface.

The author was partially supported by the Ministry of Education, Science, Sports and Culture, Japan; Grant-in-Aid for Scientific Research (B), 22340028, 2010-2014. 
Then, the Cauchy integral (1.1) of $\varphi$ has a limit at each $\zeta_{0} \in \Gamma$. Furthermore, let $F_{+}\left(\zeta_{0}\right)$ and $F_{-}\left(\zeta_{0}\right)$ denote the boundary values of $F$ from $D_{+}$and $D_{-}$, respectively, then both $F_{+}$and $F_{-}$are Hölder continuous functions of order $\alpha$ on $\Gamma$ and

$$
\begin{aligned}
& F_{+}\left(\zeta_{0}\right)=\frac{1}{2} \varphi(\zeta)+\frac{1}{2 \pi i} P . V \cdot \int_{\Gamma} \frac{\varphi(\zeta)}{\zeta-\zeta_{0}} d \zeta \\
& F_{-}\left(\zeta_{0}\right)=-\frac{1}{2} \varphi(\zeta)+\frac{1}{2 \pi i} P . V . \int_{\Gamma} \frac{\varphi(\zeta)}{\zeta-\zeta_{0}} d \zeta,
\end{aligned}
$$

where P.V. means the principal value of the integral at $\zeta_{0}$. In particular,

$$
F_{+}\left(\zeta_{0}\right)-F_{-}\left(\zeta_{0}\right)=\varphi\left(\zeta_{0}\right) .
$$

From the theorem, the principal value gives a mapping from the space of Hölder continuous functions of order $\alpha$ to itself if $\alpha \in(0,1)$ while it does not hold when $\alpha=1$ and it inspired interest in the theory of singular integrals (cf. [9]). Zygmund and I. E. Block ([3]) improved the theorem for functions of Zygmund class $\Lambda^{*}$ (see also [8] for several complex variables). Bikčantaev ([2]) generalized the theorem on open Riemann surfaces. On the other hand, J. L. Walsh ([14]) showed that the equation (1.5) holds almost everywhere if $\Gamma$ is the unit circle and $\varphi$ is in the class $L^{2}$ with respect to the Lebesgue measure $d \theta$ on the circle.

Theorem (Walsh). Let $\varphi$ be in $L^{2}(d \theta)$ on the unit circle $\left\{z=e^{i \theta}\right\}$. Then the Cauchy integral (1.1) has non-tangential limits $F_{+}\left(e^{i \theta}\right)$ and $F_{-}\left(e^{i \theta}\right)$ almost everywhere from inside and outside the circle, respectively.

In this paper, we have two purposes. The first purpose is to relax the condition (1.2) and show results similar to Plemelj-Sokthotski theorem hold. The second one is to extend Walsh's theorem for $L^{p}$ functions on boundaries of compact bordered Riemann surfaces. In the following, we will present main results in this paper. The terminologies will be given in $\S 2$.

First, we shall show an estimate of the derivative of the Cauchy integral of $\varphi$, which gives a generalization of Hardy-Littlewood theorem (cf. [12], see also [11]).

Theorem 1. Let $\Gamma$ be a smooth Jordan curve in $\mathbb{C}$ and $\varphi$ be a continuous function on $\Gamma$.

Suppose that the function $\varphi$ belongs to $\Lambda_{\Gamma}^{*}(\omega)$ for $\omega \in \mathcal{D}$. Then, there exists a constant $A>0$ such that the derivative of the Cauchy integral $F(z)$ of $\varphi$ satisfies an inequality,

$$
\left|F^{(n)}(z)\right| \delta(z)^{n} \leq A \omega(\delta(z)),
$$

for any $z \in \mathbb{C} \backslash \Gamma$ near $\Gamma$, where $\delta(z)=\operatorname{dist}(z, \Gamma):=\min _{\zeta \in \Gamma \mid}|z-\zeta|$.

Using the above theorem, we prove a generalization of Plemelj-Sokthotski theorem as a corollary. 
Corollary 1. Let $\Gamma, \varphi$ and $\omega$ be the same as in Theorem 1. Furthermore, we suppose that

$$
\int_{0}^{1} \frac{\omega(t)}{t} d t<\infty
$$

Then, the Cauchy integral $F$ of $\varphi$ has a limit at each $\zeta_{0} \in \Gamma$. Moreover, the boundary values $F_{+}\left(\zeta_{0}\right)$ and $F_{-}\left(\zeta_{0}\right)$ are given by (1.3) and (1.4).

We also give the modulus of continuity of the Cauchy integral $F$ on $\Gamma$.

Corollary 2. Let $\Gamma, \varphi$ and $\omega$ be the same as in Theorem 1. Then, the boundary functions $F_{+}$and $F_{-}$belong to $\Lambda_{\Gamma}^{*}\left(Z_{\omega}\right)$, where

$$
Z_{\omega}(t)=\max \left\{\int_{0}^{t} \omega(s) s^{-1} d s, \omega(t)\right\} \quad(t>0) .
$$

In particular,

$$
P . V . \int_{\Gamma} \frac{\varphi(\zeta)}{\zeta-\cdot} d \zeta \in \Lambda_{\Gamma}^{*}\left(Z_{\omega}\right)
$$

Remark 1. Zygmund showed that if $\Gamma$ is the unit circle and $\varphi \in \Lambda_{\Gamma}^{*}(\omega)$, then

$$
P . V . \int_{\Gamma} \frac{\varphi(\zeta)}{\zeta-\cdot} d \zeta \in \Lambda_{\Gamma}^{*}\left(Z_{\omega}^{0}\right)
$$

where

$$
Z_{\omega}^{0}(t)=\int_{0}^{t} \frac{\omega(s)}{s} d s+t \int_{t}^{t_{0}} \frac{\omega(s)}{s^{2}} d s
$$

for some $t_{0}>0$ (see [7] p. 106). See also [9] for some related results. However, it is sometimes hard to calculate $Z_{\omega}^{0}$ when $Z_{\omega}$ can be calculated (see $\S 5)$.

Finally, we consider an analogue of Walsh's theorem on compact bordered Riemann surface. Let $R$ be a compact bordered Riemann surface and $\hat{R}$ the double of $R$. Let $\hat{g}$ be the genus of $\hat{R}$. Then, there exists a canonical homology basis of $\hat{R}\left\{A_{1}, B_{1}, \ldots, A_{\hat{g}}, B_{\hat{g}}\right\}$ such that

$$
A_{i} \cap A_{j}=\emptyset, B_{i} \cap B_{j}=\emptyset \quad(i \neq j),
$$

and the intersection number of $A_{i}$ and $B_{j}$ is $\delta_{i j}$.

Take a point $\hat{P}_{0} \in \hat{R} \backslash(R \cup \partial R)$ and fix it. Let $\omega_{\hat{P}_{0}, P}$ be an abelian differential of the third kind on $\hat{R}$ with simple poles at $\hat{P}_{0}$ and $P\left(\neq \hat{P}_{0}\right)$ such that the residue at $\hat{P}_{0}$ is -1 and 1 at $P$. We assume that the differential $\omega_{\hat{P}_{0}, P}$ is normalized, that is,

$$
\int_{A_{j}} \omega_{\hat{P}_{0}, P}=0 \quad(j=1,2, \ldots, \hat{g}) .
$$


For $\varphi \in L^{1}(\partial R)$, we define a generalized Cauchy integral $F$ of $\varphi$ by

$$
F(P)=\frac{1}{2 \pi i} \int_{\partial R} \varphi \omega_{\hat{P}_{0}, P} \quad(P \notin \partial R) .
$$

Then, we have the following theorem which extends theorems of PlemeljSokthotski and Walsh. The proof will be given in $\S 7$.

Theorem 2. Let $R$ be a compact Riemann surface and $\omega_{\hat{P}_{0}, P}$ the normalized abelian differential of the third kind with poles at $\hat{P}_{0}$ and $P$ as above.

(1) If $\varphi \in L^{1}(\partial R)$, then the generalized Cauchy integral (1.9) is holomorphic on $\hat{R} \backslash \partial R$.

(2) The same statements of Theorem 1 and Corollary 1 hold for $F(P)$.

(3) Let $\varphi$ be in $L^{1}(\partial R)$. Then, the generalized Cauchy integral (1.9) of $\varphi$ has non-tangential limits almost everywhere on $\partial R$. Furthermore, if $\varphi \in L^{p}(\partial R)$ for $p>1$, the equation (1.5) holds almost everywhere on $\partial R$. Namely, let $F_{+}$and $F_{-}$denote the boundary functions from $R$ and from $\hat{R} \backslash(R \cup \partial R)$, respectively. Then,

$$
F_{+}-F_{-}=\varphi
$$

almost everywhere on $\partial R$.

Acknowledgement. The author thanks Professor Keinchi Sakan for his valuable comments.

\section{Preliminaries.}

2.1. Modulus of continuity. Let $\omega$ be a continuous function on $[0, \infty)$. We denote by $\mathcal{D}$ the set of all $\omega$ satisfying the following conditions.

(1) $\omega(0)=0$, and it is an increasing function on $[0, \infty)$.

(2) $\omega(t)$ is doubling, i. e. there exists a constant $A>0$ such that

$$
\omega(s) \leq \omega(t) \leq A \omega(s),
$$

if $0<s<t \leq 2 s$.

(3) For any $\alpha(0<\alpha<1), t^{\alpha} \leq \omega(t)$ if $t>0$ is less than some $\delta>0$.

It is easy to see that $\omega_{\alpha}(t)=\min \left\{|\log t|^{-\alpha}, A\right\}(\alpha>0, A>0)$ satisfies the above conditions.

For a continuous function $\omega$ on $[0, \infty)$, we say that a function $\varphi$ on $\Gamma$ has the modulus of continuity $\omega$ if there exists a constant $A>0$ such that

$$
\left|\varphi\left(\zeta_{1}\right)-\varphi\left(\zeta_{2}\right)\right| \leq A \omega\left(\left|\zeta_{1}-\zeta_{2}\right|\right),
$$

for every $\zeta_{1}, \zeta_{2} \in \Gamma$. We denote by $\Lambda_{\Gamma}^{*}(\omega)$ the set of functions on $\Gamma$ which have the modulus of continuity $\omega$. 
2.2. Compact bordered Riemann surfaces. Let $R$ be an open Riemann surface. A holomorphic function $f$ on $R$ is said to be of class $H^{p}(R)$ $(1 \leq p<\infty)$ if there exists a harmonic function $u$ on $R$ such that $|f|^{p} \leq u$. For $p=\infty, H^{\infty}(R)$ is the space of bounded holomorphic functions on $R$. The space $H^{p}(R)$ is called Hardy space on $R$.

We say that the Riemann surface is compact bordered if there exists a closed Riemann surface $R_{0}$ such that $R$ is a subdomain of $R_{0}$ bounded by a finite number of analytic Jordan curves. A compact bordered Riemann surface $R$ is called of type $(g, n)$ if the genus of $R$ is $g$ and the number of boundary components is $n$.

For each compact bordered Riemann surface $R$, we may consider the double of $R$. We denote it by $\hat{R}$ and by $\pi: \hat{R} \rightarrow \hat{R}$ the anticonformal involution of $\hat{R}$. If $R$ is of type $(g, n)$, then $\hat{R}$ is a closed Riemann surface of genus $2 g+n-1$.

Let $\left\{C_{1}, C_{2}, \ldots, C_{n}\right\}$ be the set of boundary curves of a compact bordered Riemann surface $R$ of type $(g, n)$. For each $C_{i}(i=1,2, \ldots, n)$ there exists an annular domain $U_{i}$ in $R$ such that $\partial U_{i}=C_{i} \cup \gamma_{i}$, where $\gamma_{i}$ is a smooth curve parallel to $C_{i}$. Then there exists a conformal mapping $f_{i}: U_{i} \cup \partial U_{i} \rightarrow$ $A_{i}:=\left\{0<r_{i} \leq|z| \leq 1\right\}$ for some $r_{i}$ such that $f_{i}\left(C_{i}\right)=\{|z|=1\}$. We say that a function $F$ on $R$ has a non-tangential limit at $p \in C_{i}$ if $F \circ f_{i}^{-1}$ has a non-tangential limit at $f_{i}(p)$, and that $F$ has non-tangential limits almost everywhere on $\partial R$ if $F \circ f_{i}^{-1}$ does so on $\{|z|=1\}$ for all $i(1=1,2, \ldots, n)$. It is not hard to see that those notions do not depend on the choice of the annular domain $U_{i}$ and the conformal mapping $f_{i}$.

We define function spaces on $\partial R$ by using $f_{i}$.

Definition 1. For $p \geq 1$, we define $L^{p}(\partial R)$ by the set of all functions $\varphi$ on $\partial R$ so that $\varphi \circ f_{i}^{-1}(i=1,2, \ldots, n)$ belong to $L^{p}$ space on the unit circle with respect to the Lebesgue measure on the circle.

Definition 2. A function $\varphi$ on $\partial R$ is said to be a Hölder continuous function of order $\alpha$ if $\varphi \circ f_{i}^{-1}(i=1,2, \ldots, n)$ are Hölder continuous functions of order $\alpha$ on the unit circle. For $\omega \in \mathcal{D}$, we denote by $\Lambda_{\partial R}^{*}(\omega)$ the set of all functions $\varphi$ on $\partial R$ such that $\varphi \circ f_{i}^{-1} \in \Lambda_{\{|z|=1\}}^{*}(\omega)(i=1,2, \ldots, n)$.

Definition 3. Let $\psi_{P}$ be an abelian differential on a neighborhood $\partial R$ with a pole at $P \in C_{i}$. For a continuous function $\varphi$ on $\partial R$, we define the principal value P. V. $\int_{\partial R} \varphi \psi_{P}$ at $P$ by

$$
\sum_{j \neq i} \int_{C_{j}} \varphi \psi_{P}+\mathrm{P} . \mathrm{V} \cdot \int_{|z|=1}\left(\varphi \circ f_{i}^{-1}\right) \psi_{P} \circ f_{i}^{-1} .
$$

Definitions 1 and 2 do not depend on the choice of $U_{i}$ and $f_{i}$ while Definition 3 may depend on them.

Here, we note the following on the boundary values of $H^{p}$-functions. 
Proposition 1. Let $R$ be a compact bordered Riemann surface. Then, every $f \in H^{p}(R)(1 \leq p \leq \infty)$ has non-tangential limits almost everywhere on $\partial R$ and the boundary function belongs to $L^{p}(\partial R)$.

From the proposition, the set of non-tangential boundary functions of $H^{p}(R)$, which we denote by $\left.H^{p}(R)\right|_{\partial R}$, is regarded as a subspace of $L^{p}(\partial R)$. M. Heins clarifies the relationship between $H^{p}(R)$ and $L^{p}(\partial R)$. To describe the result, we consider Green's function $g\left(\cdot, p_{0}\right)$ of $R$ with pole at $p_{0} \in R$. It is a positive harmonic function on $R \backslash\left\{p_{0}\right\}$ with logarithmic singularity at $p_{0}$ and vanishes identically on $\partial R$. Hence, it is extended to the double $\hat{R}$ of $R$ by $g\left(p, p_{0}\right)=-g\left(\pi(p), p_{0}\right)$ for $p \in \hat{R} \backslash R$, and $\omega_{p_{0}}:=-d g\left(\cdot, p_{0}\right)-i^{*} d g\left(\cdot, p_{0}\right)$ defines an abelian differential of the third kind on $\hat{R}$, where ${ }^{*} \psi$ stands for the conjugate differential of a differential $\psi$. The abelian differential $\omega_{p_{0}}$ has the simple poles at $p_{0}$ and $\hat{p}_{0}:=\pi\left(p_{0}\right)$, where the residues are 1 and -1 , respectively. We denote by $\delta$ the devisor of $\omega_{P_{0}}$ in $\hat{R}$. Then, Heins ([10]) shows the following:

Proposition 2. For $p \in(1, \infty)$, the decomposition

$$
L^{p}(\partial R)=\left.H^{p}(R)\right|_{\partial R}+\left.\overline{H_{0}^{p}(R)}\right|_{\partial R}+\left.M\left(\delta^{-1}\right)\right|_{\partial R}
$$

holds, where $H_{0}^{p}(R)$ is the space of holomorphic functions in $H^{p}(R)$ which vanish at $P_{0}$ and $M\left(\delta^{-1}\right)$ is the space of meromorphic functions on $\hat{R}$ whose devisors are multiple of $\delta^{-1}$.

3. Proof of Theorem 1. Let $z_{0}$ be a point in $\mathbb{C} \backslash \Gamma$ and $\zeta_{0} \in \Gamma$ a point with $\delta\left(z_{0}\right)=\left|z_{0}-\zeta_{0}\right|$. We take an interval $I\left(z_{0}, \zeta_{0}\right)$ whose end points are $z_{0}$ and $\zeta_{0}$. Then, for any $z \in I\left(z_{0}, \zeta_{0}\right),\left|z-\zeta_{0}\right|=\delta(z)$. Namely, $\zeta_{0}$ is the nearest point of $\Gamma$ from $z$.

Since

$$
F^{\prime}(z)=\frac{1}{2 \pi i} \int_{\Gamma} \frac{\varphi(\zeta)-\varphi\left(\zeta_{0}\right)}{(\zeta-z)^{2}} d \zeta
$$

we have

$$
\left|F^{(n)}(z)\right| \leq \frac{n !}{2 \pi} \int_{\Gamma} \frac{\left|\varphi(\zeta)-\varphi\left(\zeta_{0}\right)\right|}{|\zeta-z|^{n+1}}|d \zeta|
$$

Since $\zeta=\zeta(s)$ is differentiable, there exists $\delta>0$ such that for each $t \in$ $[-\delta, \delta]$ there exists a point $\zeta_{t} \in \Gamma$ such that $\arg \left(\zeta_{t}-z_{0}\right)-\arg \left(\zeta_{0}-z_{0}\right)=t$ and the segment $I_{t}$ between $z_{0}$ and $\zeta_{t}$ intersects only at $\zeta_{t}$ with $\Gamma$. It suffices to show that (1.6) is valid in a neighborhood of $\Gamma$. Hence, we may assume that there exists a constant $\varepsilon_{0}>0$ such that $\delta, \delta\left(z_{0}\right)>\varepsilon_{0}$. 
Then, for $z \in I_{0} \subset I\left(z_{0}, \zeta_{0}\right)$ we have

$$
\begin{aligned}
\left|F^{(n)}(z)\right| & \leq \frac{n !}{2 \pi} \int_{\Gamma} \frac{\left|\varphi(\zeta)-\varphi\left(\zeta_{0}\right)\right|}{|\zeta-z|^{n+1}}|d \zeta| \\
& =\frac{n !}{2 \pi} \int_{\Gamma_{\delta}} \frac{\left|\varphi(\zeta)-\varphi\left(\zeta_{0}\right)\right|}{|\zeta-z|^{n+1}}|d \zeta|+\frac{n !}{2 \pi} \int_{\Gamma \backslash \Gamma_{\delta}} \frac{\left|\varphi(\zeta)-\varphi\left(\zeta_{0}\right)\right|}{|\zeta-z|^{n+1}}|d \zeta| \\
& =: A_{1}(z)+A_{2}(z),
\end{aligned}
$$

where $\Gamma_{\delta} \ni \zeta_{0}$ is the subarc of $\Gamma$ between $\zeta_{-\delta}$ and $\zeta_{\delta}$.

Now, we consider the behavior of $\left|F^{(n)}(z)\right|$ as $I_{0} \ni z \rightarrow \zeta_{0}$. We may assume that $\left|z-z_{0}\right|>\delta\left(z_{0}\right) / 2$.

Since the point $z$ lies in a neighborhood of $\Gamma$, by taking a sufficiently small $\delta>0$ if necessary, we suppose that

$$
\left|d \zeta_{t}\right| \leq 2 d t
$$

on $\Gamma_{\delta}$.

There is a point $z_{t}$ on $I_{t}$ with $\left|z_{t}-z_{0}\right|=\delta\left(z_{0}\right) \leq\left|\zeta_{t}-z_{0}\right|$ for each $t \in[-\delta, \delta]$. Thus, we have

$$
\begin{aligned}
A_{1}(z) & \leq \frac{n !}{2 \pi} \int_{\Gamma_{\delta}} \frac{\left|\varphi(\zeta)-\varphi\left(\zeta_{0}\right)\right|}{\left|z_{t}-z\right|^{n+1}}|d \zeta| \\
& \leq \frac{1}{\pi} \int_{-\delta}^{\delta} \frac{\left|\varphi\left(\zeta_{t}\right)-\varphi\left(\zeta_{0}\right)\right|}{\left|z_{t}-z\right|^{n+1}} d t \\
& \leq \frac{A n !}{\pi \delta\left(z_{0}\right)^{n+1}} \int_{-\delta}^{\delta} \frac{\omega\left(\left|\zeta_{t}-\zeta_{0}\right|\right)}{\left(1-2 r \cos t+r^{2}\right)^{(n+1) / 2}} d t
\end{aligned}
$$

where $r=\left|z-z_{0}\right| \delta\left(z_{0}\right)^{-1} \in\left(\frac{1}{2}, 1\right)$. Noting that

$$
1-2 r \cos t+r^{2} \geq(1-r)^{2}+\frac{4 r t^{2}}{\pi^{2}},
$$

we have

$$
A_{1}(z) \leq \frac{A n !}{2 \pi \delta\left(z_{0}\right)^{n+1}} \int_{-\delta}^{\delta} \frac{\omega(|t|)}{\left\{(1-r)^{2}+4 r(t / \pi)^{2}\right\}^{(n+1) / 2}} d t .
$$

Setting $C_{r}=\pi^{2}(1-r)^{2} / 4 r$ and $t=\sqrt{C_{r}} \tan \theta$, we have

$$
\begin{aligned}
B(z) & :=\frac{A n !}{2 \pi \delta\left(z_{0}\right)^{n+1}} \int_{-\delta}^{\delta} \frac{\omega(|t|)}{\left\{(1-r)^{2}+4 r(t / \pi)^{2}\right\}^{(n+1) / 2}} d t \\
& =\frac{A \pi^{2} n !}{4 r \delta\left(z_{0}\right)^{n+1}} \int_{-\delta}^{\delta} \frac{\omega(|t|)}{\left\{t^{2}+C_{r}\right\}^{(n+1) / 2}} d t
\end{aligned}
$$




$$
\begin{aligned}
& =\frac{A \pi^{2} n !}{4 r \delta\left(z_{0}\right)^{n+1}\left(\sqrt{C_{r}}\right)^{n}} \int_{-\beta_{r}}^{\beta_{r}} \cos \theta^{n-1} \omega\left(\left|\sqrt{C_{r}} \tan \theta\right|\right) d \theta \\
& \leq \frac{A \pi^{2} n !}{2 r \delta\left(z_{0}\right)^{n+1}\left(\sqrt{C_{r}}\right)^{n}} \int_{0}^{\beta_{r}} \omega\left(\left|\sqrt{C_{r}} \tan \theta\right|\right) d \theta,
\end{aligned}
$$

where $\beta_{r}=\arctan \frac{\delta}{\sqrt{C_{r}}} \in(0, \pi / 2)$. As $z \rightarrow \zeta_{0}, r \rightarrow 1, C_{r} \rightarrow 0$ and $\beta_{r} \rightarrow \pi / 2$. We take $z \in I_{0}$ sufficiently close to $\zeta_{0}$ so that $C_{r}<1$.

When $\theta \in\left(0, \frac{\pi}{4}\right], \tan \theta \in(0,1]$. Hence, $\left|\sqrt{C_{r}} \tan \theta\right| \leq \sqrt{C_{r}}$ and $\omega\left(\left|\sqrt{C_{r}} \tan \theta\right|\right) \leq \omega\left(\sqrt{C_{r}}\right)$. Thus, we have

$$
\begin{aligned}
\int_{0}^{\pi / 4} \omega\left(\left|\sqrt{C_{r}} \tan \theta\right|\right) d \theta & \leq \int_{0}^{\pi / 4} \omega\left(\sqrt{C_{r}}\right) d \theta \\
& =\frac{\pi}{4} \omega\left(\sqrt{C_{r}}\right),
\end{aligned}
$$

and

$$
\frac{1}{\left(\sqrt{C_{r}}\right)^{n}} \int_{0}^{\pi / 4} \omega\left(\left|\sqrt{C_{r}} \tan \theta\right|\right) d \theta \leq \frac{\pi}{4\left(\sqrt{C_{r}}\right)^{n}} \omega\left(\sqrt{C_{r}}\right) .
$$

For any $\sigma \in(0,1)$, we put $\lambda=1-\sigma \in(0,1)$ and $\gamma_{r}:=\arctan \left(\frac{1}{\sqrt{C_{r}}}\right)^{\lambda}$. We may assume that $\gamma_{r}<\beta_{r}$. When $\theta \in\left(\frac{\pi}{4}, \gamma_{r}\right], \tan \theta \in\left(1, C_{r}^{-\lambda / 2}\right]$ and we have

Therefore,

$$
\omega\left(\sqrt{C_{r}} \tan \theta\right) \leq \omega\left(\sqrt{C_{r}^{1-\lambda}}\right)
$$

$$
\int_{\pi / 4}^{\gamma_{r}} \omega\left(\sqrt{C_{r}} \tan \theta\right) d \theta \leq \frac{\pi}{4} \omega\left(\sqrt{C_{r}^{1-\lambda}}\right)
$$

and

$$
\frac{1}{\left(\sqrt{C_{r}}\right)^{n}} \int_{\pi / 4}^{\gamma_{r}} \omega\left(\left|\sqrt{C_{r}} \tan \theta\right|\right) d \theta \leq \frac{\pi}{4\left(\sqrt{C_{r}}\right)^{n}} \omega\left(\sqrt{C_{r}^{1-\lambda}}\right) .
$$

Finally, we consider the case where $\theta \in\left(\gamma_{r}, \beta_{r}\right]$. Since $\arctan x=$ $\int_{0}^{x} \frac{1}{1+t^{2}} d t$, we have

$$
\beta_{r}-\gamma_{r}=\int_{\left(\sqrt{C_{r}}\right)^{-\lambda}}^{\delta / \sqrt{C_{r}}} \frac{d x}{x^{2}+1} \leq \int_{\left(\sqrt{C_{r}}\right)^{-\lambda}}^{\delta / \sqrt{C_{r}}} \frac{d x}{x^{2}} \leq A_{1} \sqrt{C_{r}^{\lambda}},
$$

where the constant $A_{1}>0$ depends only on $r$, and $A_{1} \rightarrow 1$ as $r \rightarrow 1$. On the other hand,

and

$$
\left(\frac{1}{\sqrt{C_{r}}}\right)^{-\lambda} \leq \tan \theta \leq\left(\frac{\delta}{\sqrt{C_{r}}}\right)
$$

$$
\omega\left(\sqrt{C_{r}} \tan \theta\right) \leq \omega(\delta) .
$$


Therefore, we conclude

$$
\frac{1}{\left(\sqrt{C_{r}}\right)^{n}} \int_{\gamma_{r}}^{\beta_{r}} \omega\left(\left|\sqrt{C_{r}} \tan \theta\right|\right) d \theta \leq \frac{A_{1} \sqrt{C_{r}^{\lambda}}}{\left(\sqrt{C_{r}}\right)^{n}} \omega(\delta) .
$$

Combining (3.2), (3.3) and (3.4), we have

$$
B(z) \leq \frac{A_{2}}{\left(\sqrt{C_{r}}\right)^{n}}\left\{\omega\left(\sqrt{C_{r}}\right)+\omega\left(\sqrt{C_{r}^{1-\lambda}}\right)+\sqrt{C_{r}^{\lambda}} \omega(\delta)\right\},
$$

for some constant $A_{2}$ depending only on $r$. Since $0<1-\lambda<1$, we have $\omega\left(\sqrt{C_{r}^{1-\lambda}}\right) \geq \omega\left(\sqrt{C_{r}}\right)$ as $C_{r} \rightarrow 0$. By the definition of $\mathcal{D}, \sqrt{C_{r}^{\lambda}} \leq \omega\left(\sqrt{C_{r}}\right)$. Hence,

$$
B(z) \leq \frac{A_{3}}{\left(\sqrt{C_{r}}\right)^{n}} \omega\left(\sqrt{C_{r}^{1-\lambda}}\right),
$$

where the constant $A_{3}>0$ depends only on $r<1$ and $\delta>0$, and it is bounded if $r$ is sufficiently close to 1 and $\delta$ is sufficiently small.

Noting that $\delta\left(z_{0}\right)>\varepsilon, 1-r=\left(\delta\left(z_{0}\right)-\left|z-z_{0}\right|\right) \delta\left(z_{0}\right)^{-1}=\delta(z) \delta\left(z_{0}\right)^{-1}$ and

$$
\frac{\pi^{2}}{4}(1-r)^{2} \leq C_{r} \leq \frac{\pi^{2}}{2}(1-r)^{2}
$$

we obtain, for $\sigma=1-\lambda \in(0,1)$,

$$
A_{1}(z) \leq B(z) \leq \frac{A_{3}}{\delta(z)^{n}} \omega\left(\left(\varepsilon^{-1} \pi \delta(z)\right)^{\sigma}\right) \leq \frac{A_{3} A^{\log \varepsilon^{-1} \pi / \log 2}}{\delta(z)^{n}} \omega\left(\delta(z)^{\sigma}\right),
$$

by the doubling property of $\omega$. Here, $A$ is the constant of the doubling property of $\omega$.

Since $\delta>\varepsilon$, it is not hard to see that $A_{2}(z)$ is bounded by some constant $M>0$ as $z \rightarrow \zeta_{0}$. Hence, we have

$$
\left|A_{2}(z)\right| \leq M=\frac{M}{\delta(z)^{n}} \delta(z)^{n} \leq \frac{M}{\delta(z)^{n}} \omega\left(\delta(z)^{\sigma}\right) .
$$

if $\delta(z)>0$ is sufficiently small. Therefore, we conclude that there exists a constant $A_{5}>0$ such that

$$
\left|F^{(n)}(z)\right| \delta(z)^{n} \leq A_{5} \omega\left(\delta(z)^{\sigma}\right) .
$$

Since the constant $A_{5}$ is independent of $\sigma$, we have

$$
\left|F^{(n)}(z)\right| \delta(z)^{n} \leq A_{5} \omega(\delta(z)) .
$$

By using the differentiability, we see that we can take $\varepsilon>0$ sufficiently small so that there exists a neighborhood $U$ of $\Gamma$ such that for any $z \in U \backslash \Gamma$, there exist points $z_{0}, \zeta_{0}$ satisfying the above conditions. Thus, we verify that there (3.5) holds for any $z \in \mathbb{C} \backslash \Gamma$ near $\Gamma$. 
Remark 2. By using a similar argument, we extend the Hardy-Littlewood theorem on the unit disk which gives an estimate of the derivatives of holomorphic functions on the disk and continuous on the closed disk ([12]). Recently, H. Aikawa ([1]) estimates the norms of gradient vectors of harmonic functions on certain domains in $\mathbb{R}^{n}$.

4. Proof of Corollary 1. At first, we shall show that the principal value of the integral exists. Let $\zeta=\zeta(s)$ be the arc-length parametrization of $\Gamma$ with $\zeta(0)=\zeta_{0}$. Since $\zeta(s)$ is differentiable, there exists a constant $A>0$ such that

$$
A^{-1}|s| \leq\left|\zeta(s)-\zeta_{0}\right| \leq A|s|
$$

holds near $t=0$. From the doubling condition of $\omega$ and (2.1), we have

$$
\left|\varphi(\zeta(s))-\varphi\left(\zeta_{0}\right)\right| \leq A \omega(|s|),
$$

near $s=0$. Hence, it follows from (1.7) that the principal value P.V. $\int_{\Gamma} \varphi(\zeta) d \zeta /\left(\zeta-\zeta_{0}\right)$ exists.

Next, we consider the existence of the boundary value $F_{+}\left(\zeta_{0}\right)$ of $F(z)$. For each $\zeta_{0} \in \Gamma$, we may take a point $z_{0} \in D_{+}$so that $\delta\left(z_{0}\right)=\left|z_{0}-\zeta_{0}\right|$. Let $I\left(z_{0}, \zeta_{0}\right)$ denote the interval between $z_{0}$ and $\zeta_{0}$ and take two points $z, z^{\prime}$ on $I\left(z_{0}, \zeta_{0}\right)$ so that $\delta(z) \geq \delta\left(z^{\prime}\right)>0$. It follows from Theorem 1 that for any $\sigma \in(0,1)$

$$
\begin{aligned}
\left|F(z)-F\left(z^{\prime}\right)\right| & =\left|\int_{z^{\prime}}^{z} F^{\prime}(z) d z\right| \\
& \leq \int_{z^{\prime}}^{z}\left|F^{\prime}(z)\right||d z| \\
& \leq A \int_{\delta\left(z^{\prime}\right)}^{\delta(z)} \frac{\omega\left(t^{\sigma}\right)}{t} d t \quad\left(s=t^{\sigma}\right) \\
& \leq \frac{1}{\sigma} \int_{\delta\left(z^{\prime}\right)^{1 / \sigma}}^{\delta(z)^{1 / \sigma}} \frac{\omega(s)}{s} d s .
\end{aligned}
$$

From (1.7), we verify that $\lim _{z \rightarrow \zeta_{0}} F(z)$ exists along $I\left(z_{0}, \zeta_{0}\right)$ and the convergence is uniform. Hence, the boundary function is continuous and it guarantees the existence of $F_{+}\left(\zeta_{0}\right)$. By the same proof, we can show the existence of $F_{-}\left(\zeta_{0}\right)$.

Finally, we show (1.3) and (1.4). In fact, a standard argument gives the proof from the existence of $F_{+}$and $F_{-}$(cf. [6]). We shall give the proof for convenience of the reader.

For $\delta>0$, we put $\Gamma(\delta)=\left\{\left|z-\zeta_{0}\right|<\delta\right\} \cap \Gamma$ and

$$
\Phi(z)=\frac{1}{2 \pi i} \int_{\Gamma} \frac{\varphi(\zeta)-\varphi\left(\zeta_{0}\right)}{\zeta-z} d \zeta,
$$


for $z \in D_{+}$. Then,

$$
\begin{aligned}
\Phi(z) & -\frac{1}{2 \pi i} P . V . \int_{\Gamma} \frac{\varphi(\zeta)-\varphi\left(\zeta_{0}\right)}{\zeta-\zeta_{0}} d \zeta \\
& =\frac{z-\zeta_{0}}{2 \pi i} P . V . \int_{\Gamma} \frac{\varphi(\zeta)-\varphi\left(\zeta_{0}\right)}{\left(\zeta-\zeta_{0}\right)(\zeta-z)} d \zeta \\
& =\frac{z-\zeta_{0}}{2 \pi i} P . V . \int_{\Gamma(\delta)} \frac{\varphi(\zeta)-\varphi\left(\zeta_{0}\right)}{\left(\zeta-\zeta_{0}\right)(\zeta-z)} d \zeta \\
& +\frac{z-\zeta_{0}}{2 \pi i} \int_{\Gamma \backslash \Gamma(\delta)} \frac{\varphi(\zeta)-\varphi\left(\zeta_{0}\right)}{\left(\zeta-\zeta_{0}\right)(\zeta-z)} d \zeta \\
& :=I_{1}(z)+I_{2}(z) .
\end{aligned}
$$

We have already seen that the boundary value $F_{+}\left(\zeta_{0}\right)$ exists. Therefore, we may assume that $z \in D_{+}$approach to $\zeta_{0}$ along $I\left(z_{0}, \zeta_{0}\right)$. It is not hard to see that there exists a constant $A>0$ such that for and $z \in I\left(z_{0}, \zeta_{0}\right)$ for any $\zeta \in \Gamma(\delta)$, an inequality

$$
|z-\zeta| \geq A\left|z-\zeta_{0}\right|
$$

holds. Hence, we have

$$
\begin{aligned}
\left|I_{1}(z)\right| & \leq \frac{\left|z-\zeta_{0}\right|}{2 \pi} P . V . \int_{\Gamma(\delta)} \frac{\left|\varphi(\zeta)-\varphi\left(\zeta_{0}\right)\right|}{\left|\left(\zeta-\zeta_{0}\right)(\zeta-z)\right|}|d \zeta| \\
& \leq \frac{A}{2 \pi} P . V . \int_{\Gamma(\delta)} \frac{\left|\varphi(\zeta)-\varphi\left(\zeta_{0}\right)\right|}{\left|\left(\zeta-\zeta_{0}\right)\right|}|d \zeta| \\
& \leq \frac{A}{2 \pi} P . V . \int_{\Gamma(\delta)} \frac{\omega\left(\left|\zeta-\zeta_{0}\right|\right)}{\left|\left(\zeta-\zeta_{0}\right)\right|}|d \zeta| .
\end{aligned}
$$

Thus, for arbitrary small $\varepsilon>0$ it follows from (1.7) that $\left|I_{1}(z)\right|<\varepsilon$ if $\delta>0$ is sufficiently small.

On the other hand, $\lim _{z \rightarrow \zeta_{0}} I_{2}(z)=0$ and we have

$$
\lim _{z \rightarrow \zeta_{0}} \Phi(z)=\frac{1}{2 \pi i} P . V \cdot \int_{\Gamma} \frac{\varphi(\zeta)-\varphi\left(\zeta_{0}\right)}{\zeta-\zeta_{0}} d \zeta .
$$

Noting that

$$
\frac{1}{2 \pi i} P . V . \int_{\Gamma} \frac{1}{\zeta-\zeta_{0}} d \zeta=\frac{1}{2}
$$

we obtain

$$
F_{+}\left(\zeta_{0}\right)-\varphi\left(\zeta_{0}\right)=\frac{1}{2 \pi i} P . V . \int_{\Gamma} \frac{\varphi(\zeta)}{\zeta-\zeta_{0}} d \zeta-\frac{1}{2} \varphi\left(\zeta_{0}\right)
$$

and it shows (1.3) as desired. The proof of (1.4) is the same. 
5. Proof of Corollary 2. Let $\zeta_{1}, \zeta_{2}$ be on $\Gamma$. Then, it follows from Theorem 1 that

$$
\begin{aligned}
\left|F_{+}\left(\zeta_{1}\right)-F_{+}\left(\zeta_{2}\right)\right| & =\left|\int_{I\left(z_{1}, \zeta_{1}\right)+\gamma+I\left(z_{2}, \zeta_{2}\right)} F^{\prime}(z) d z\right| \\
& \leq A \int_{I\left(z_{1}, \zeta_{1}\right)+\gamma+I\left(z_{2}, \zeta_{2}\right)} \frac{\omega(\delta(z))}{\delta(z)}|d z|,
\end{aligned}
$$

where $z_{j} \in D_{+}(j=1,2)$ are in a neighborhood fo $\zeta_{j}$ and $\gamma \subset D_{+}$is a smooth arc connecting $z_{1}$ and $z_{2}$. We may take $\zeta_{1}$ and $\zeta_{2}$ sufficiently close to each other so that the length of $\gamma$ is less than $A\left|\zeta_{1}-\zeta_{2}\right|$ and

$$
A^{-1} \delta\left(z_{1}\right) \leq \delta(z) \leq A \delta\left(z_{1}\right)
$$

for any $z \in \gamma$. We may also take $z_{j}$ so that the length of $I\left(z_{j}, \zeta_{j}\right)$ is $\delta\left(z_{j}\right)$ $(j=1,2)$. Then, we have

$$
\int_{I\left(z_{j}, \zeta_{j}\right)} \frac{\omega(\delta(z))}{\delta(z)}|d z|=\int_{0}^{\delta\left(z_{j}\right)} \frac{\omega(t)}{t} d t \quad(j=1,2) .
$$

As for the integral along $\gamma$, from (5.1) and the doubling property of $\omega$, we obtain

$$
\int_{\gamma} \frac{\omega(\delta(z))}{\delta(z)}|d z| \leq A \int_{\gamma} \frac{\omega\left(\delta\left(z_{1}\right)\right)}{\delta\left(z_{1}\right)}|d z| \leq A^{\prime} \frac{\omega\left(\delta\left(z_{1}\right)\right)}{\delta\left(z_{1}\right)}\left|\zeta_{1}-\zeta_{2}\right| .
$$

Therefore, by taking $\delta\left(z_{1}\right)=\left|\zeta_{1}-\zeta_{2}\right|$, we obtain

$$
\left|F_{+}\left(\zeta_{1}\right)-F_{+}\left(\zeta_{2}\right)\right| \leq A Z_{\omega}\left(\left|\zeta_{1}-\zeta_{2}\right|\right),
$$

and the proof is completed.

6. Examples. In this section, we shall give examples for our theorems.

Example 1. We have seen that $\omega_{\alpha}(t)=\min \left\{|\log t|^{-\alpha}, A\right\}(\alpha>0)$ belongs to $\mathcal{D}$. Therefore, from Theorem 1 we have

$$
\left|F^{(n)}(z) \delta(z)^{n}\right| \leq A|\log \delta(z)|^{-\alpha},
$$

for $\varphi \in \Lambda_{\Gamma}^{*}\left(\omega_{\alpha}\right)$.

We also see that

$$
\int_{0}^{\delta} \frac{\omega_{\alpha}(t)}{t} d t<\infty
$$

for small $\delta>0$ if and only if $\alpha>1$. Hence, from Corollary 1, if $\alpha>1$, then

$$
P . V . \int_{\Gamma} \frac{\varphi(\zeta)}{\zeta-\cdot} d \zeta \in \Lambda_{\Gamma}^{*}\left(\omega_{\alpha-1}\right) .
$$

Now, we consider Zygmund's estimate $Z_{\omega_{\alpha}}^{0}$ given by (1.8). For $\omega=\omega_{\alpha}$ $(\alpha>1)$, it is not hard to see that

$$
t \int_{t}^{t_{0}} \frac{\omega_{\alpha}(s)}{s^{2}} d s \leq \omega_{\alpha}(t)
$$


Example 2. Put $\hat{\omega}_{\alpha}(t)=\min \left\{|\log t|^{-1}|\log | \log t||^{-\alpha}, A\right\} \quad(\alpha>0)$. Then, we see that $\hat{\omega}_{\alpha} \in \mathcal{D}$. Hence, for $\varphi \in \Lambda_{\Gamma}^{*}\left(\hat{\omega}_{\alpha}\right)$, Theorem 1 gives

$$
\left|F^{(n)}(z) \delta(z)^{n}\right| \leq A|\log \delta(z)|^{-1}|\log | \log \delta(z)||^{-\alpha} .
$$

It is easily seen that

$$
\int_{0}^{\delta} \frac{\hat{\omega}_{\alpha}(t)}{t} d t<\infty
$$

if and only if $\alpha>1$. Therefore, we see that if $\alpha>1$, then

$$
P . V . \int_{\Gamma} \frac{\varphi(\zeta)}{\zeta-\cdot} d \zeta \in \Lambda_{\Gamma}^{*}\left(|\log | \log t||^{1-\alpha}\right) .
$$

More generally, we may consider $\hat{\omega}_{n, \alpha}(t)=\prod_{k=1}^{n-1} f_{k}(t)^{-1} f_{n}(t)^{-\alpha}(t)(\alpha>0)$, where $f_{1}(t)=|\log t|$ and $f_{n+1}(t)=f_{n}\left(f_{1}(t)\right)$. Then, $\hat{\omega}_{n, \alpha} \in \mathcal{D}$.

By putting $x=f_{n}(s)$, we get

$$
\int_{0}^{t} \frac{\hat{\omega}_{n, \alpha}(s)}{s} d s=\int_{f_{n}(t)}^{\infty} x^{-\alpha} d x
$$

for sufficiently small $t>0$. Therefore, we obtain that if $\varphi \in \Lambda_{\Gamma}^{*}\left(\hat{\omega}_{n, \alpha}\right)$ for $\alpha>1$, then

$$
P . V . \int_{\Gamma} \frac{\varphi(\zeta)}{\zeta-.} d \zeta \in \Lambda_{\Gamma}^{*}\left(f_{n}(t)^{1-\alpha}\right) .
$$

On the other hand, if we consider Zygmund's estimate (1.8), we have to estimate the integral

$$
t \int_{t}^{t_{0}} \frac{\hat{\omega}_{n, \alpha}(s)}{s^{2}} d s=t \int_{f_{n}\left(t_{0}\right)}^{f_{n}(t)} \frac{1}{f_{n}^{-1}(x)} d x .
$$

as $t \rightarrow 0$. At least for the author, it is much harder than the above one.

7. Proof of Theorem 2. First, we consider the behavior of sequences of abelian differentials on closed Riemann surfaces.

Let $X$ be a closed Riemann surface of genus $g$ and $\left\{P_{1, n}\right\}_{n=1}^{\infty},\left\{P_{2, n}\right\}_{n=1}^{\infty}$ be distinct sequences on $X$ converging to a point $P_{0}$. We assume that both $P_{1, n}$ and $P_{2, n}$ are contained in a parametric disk $U$ of $P_{0}$. Let $\zeta: U \rightarrow$ $\{|\zeta|<1\}$ be a local coordinate on $U$ with $\zeta\left(P_{0}\right)=0$. Put $b_{1, n}:=\zeta\left(P_{1, n}\right)$ and $b_{2, n}:=\zeta\left(P_{2, n}\right)$.

We consider abelian differentials $\omega_{n}(n=0,1,2, \ldots)$ on $X$ satisfying the following conditions.

(1) The differentials $\omega_{n}(n=1,2, \ldots)$ are holomorphic on $X \backslash\left\{P_{1, n}, P_{2, n}\right\}$ and $\omega_{0}$ is holomorphic on $X$;

(2) For each $j(j=1,2, \ldots, g)$,

$$
\alpha_{j}(n):=\int_{A_{i}} \omega_{n} \rightarrow \int_{A_{j}} \omega_{0}:=\alpha_{j} \quad(n \rightarrow \infty),
$$


where $\left\{A_{j}, B_{j}\right\}_{j=1}^{g}$ is a canonical homology basis of $X$ defined as in $\S 2$

(3) Let

$$
\left\{\sum_{k=1}^{l_{1, n}} c_{1,-k}(n)\left(\zeta-b_{1, n}\right)^{-k}+\sum_{k=0}^{\infty} c_{1, k}(n)\left(\zeta-b_{1, n}\right)^{k}\right\} d \zeta
$$

and

$$
\left\{\sum_{k=1}^{l_{2, n}} c_{2,-k}(n)\left(\zeta-b_{2, n}\right)^{-k}+\sum_{k=0}^{\infty} c_{2, k}(n)\left(\zeta-b_{2, n}\right)^{k}\right\} d \zeta
$$

be Laurent expansions of $\omega_{n}$ at $P_{1, n}$ and $P_{2, n}$, respectively. Then, $l_{1, n}=l_{2, n}=l$ for some $l \in \mathbb{N}$ and $\lim _{n \rightarrow \infty} c_{j,-k}(n)(j=1,2)$ exist for each $k\left(k=1,2, \ldots, l_{1, n}\right)$, which satisfy

$$
\lim _{n \rightarrow \infty} c_{1,-k}(n)=-\lim _{n \rightarrow \infty} c_{2,-k}(n) .
$$

Under those conditions, we have the following.

Lemma 1. Let $Q$ be a point in $X \backslash \bigcup_{n=1}^{\infty}\left\{P_{1, n}, P_{2, n}\right\} \cup\left\{P_{0}\right\}$ and $z$ a local coordinate at $Q$ with $z(Q)=0$. If $\omega_{0}$ and $\omega_{n}$, which are holomorphic at $Q$, have expansions:

$$
\omega_{0}=\sum_{m=0}^{\infty} a_{m}(Q) z^{m} d z, \quad \omega_{n}=\sum_{m=0}^{\infty} a_{m, n}(Q) z^{m} d z
$$

with respect to $z$ at $Q$, then $\lim _{n \rightarrow \infty} a_{m, n}(Q)=a_{m}(Q)(m=0,1,2, \ldots)$. Furthermore, the convergence is locally uniform in $X \backslash \bigcup_{n=1}^{\infty}\left\{P_{1, n}, P_{2, n}\right\} \cup$ $\left\{P_{0}\right\}$.

Proof. By considering $\omega_{n}-\omega_{0}$, we may assume that $\alpha_{j}=0$ for $j \in$ $\{1,2, \ldots g\}$ and $a_{m}=0$ for any $m \in \mathbb{N} \cup\{0\}$.

Let $\psi_{m}$ be an abelian differential holomorphic on $X \backslash\{Q\}$ with pole of order $m+2$ at $Q$ which has the expansion:

$$
\psi_{m}=\left\{\frac{1}{z^{m+2}}+\text { (holomorphic) }\right\} d z
$$

with respect to the local coordinate $z$. For the abelian integral $\Psi_{m}(P)=$ $\int_{P} \psi_{m}$ on $X \backslash \bigcup_{j=1}^{g} A_{j} \cup B_{j}$, we have

$$
\begin{aligned}
2 \pi i \sum_{P \in X} \operatorname{res}_{P} \Psi_{m} \omega_{n} & =\sum_{j=1}^{g}\left[\int_{A_{j}} \psi_{m} \int_{B_{j}} \omega_{n}-\int_{B_{j}} \psi_{m} \int_{A_{j}} \omega_{n}\right] \\
& =-\sum_{j=1}^{g} \alpha_{j}(n) \int_{B_{j}} \psi_{m}
\end{aligned}
$$

from the bilinear relation (cf. [5]). 
The poles of $\Psi_{m} \omega_{n}$ are $P_{1, n}, P_{2, n}$ and $Q$. As for $Q$, from (7.3) we obtain the residue at $Q$ :

$$
\operatorname{res}_{Q} \Psi_{m} \omega_{n}=\frac{-1}{m+1} a_{m, n}(Q) .
$$

Since $\Psi_{m}$ is holomorphic at $P_{1, n}$ and $P_{2, n}$, it has expansions:

$$
\Psi_{m}(\zeta)=\sum_{k=0}^{\infty} \beta_{j, k}(n)\left(\zeta-b_{j, n}\right)^{k}
$$

at $b_{j, n}(j=1,2)$. Thus,

$$
\operatorname{res}_{P_{j, n}} \Psi_{m} \omega_{n}=\sum_{k=1}^{l} c_{j,-k}(n) \beta_{j, k-1}(n) \quad(j=1,2),
$$

and we get

$$
\begin{aligned}
& \frac{-1}{m+1} a_{m, n}(Q)+\sum_{k=1}^{l} c_{1,-k}(n) \beta_{1, k-1}(n)+\sum_{k=1}^{l} c_{2,-k}(n) \beta_{2, k-1}(n) \\
& =-\frac{1}{2 \pi i} \sum_{j=1}^{g} \alpha_{j}(n) \int_{B_{j}} \psi_{m} .
\end{aligned}
$$

We have

$$
\lim _{n \rightarrow \infty} \beta_{1, k}(n)=\lim _{n \rightarrow \infty} \beta_{2, k}(n) \quad(j=0,1, \ldots, l-1) .
$$

Therefore, from (7.2) we obtain

$$
\lim _{n \rightarrow \infty} a_{m, n}(Q)=0 .
$$

The uniform convergence is also easily shown.

Now, we proceed to prove Theorem 2.

Proof of (1). Let $\varphi$ be in $L^{1}(\partial R)$. It is known that the normalized abelian differential $\omega_{\hat{P}_{0}, P}$ is holomorphic on $X \backslash\left\{\hat{P}_{0}\right\}$ for $P$ (cf. [5] III. 3). Thus, $F(P)=\frac{1}{2 \pi i} \int_{\partial R} \varphi \omega_{\hat{P}_{0}, P}$ is holomorphic on $R \backslash \partial R \cup\left\{\hat{P}_{0}\right\}$. Furthermore, it follows from Lemma 1 that $\omega_{\hat{P}_{0}, P}$ uniformly converges to zero on $\partial R$ as $P \rightarrow \hat{P}_{0}$. We conclude that $F(P)$ is holomorphic on $\hat{R} \backslash \partial R$.

Proof of (2). The proof is done by localization. Let $Q \in C_{i}$ and $U_{i}$ an annular domain in $R$ as in $\S 2$. Then, $\hat{U}_{i}:=U_{i} \cup C_{i} \cup \pi\left(U_{i}\right)$ is an annular neighborhood of $C_{i}$ in $\hat{R}$, where $\pi: \hat{R} \rightarrow \hat{R}$ is the anti-conformal involution of $\hat{R}$. We may assume that $\hat{P}_{0} \notin \hat{U}_{i}$. There exists a conformal mapping $f: \hat{U}_{i} \rightarrow A_{r_{i}}:=\left\{0<r_{i}<|z|<r_{i}^{-1}\right\}$ such that $f\left(C_{i}\right)=\{|z|=1\}, f(Q)=1$ and $f\left(\partial \hat{U}_{i} \cap R\right)=\left\{|z|=r_{i}\right\}$. Then, a differential

$$
\theta_{P}=\omega_{\hat{P}_{0}, P} \circ f^{-1}(z)-\frac{1}{z-f(P)} d z
$$


is holomorphic in $A_{r_{i}}$. Hence, there exists a holomorphic function $h_{P}$ on $A_{r}$ such that $\theta_{P}=h_{P}(z) d z$. Because of the uniform convergence of $\omega_{\hat{P}_{0}, P} \circ f^{-1}$ on $\partial A_{r_{i}}$ as $P \rightarrow Q$, holomorphic functions $h_{P}$ converges to $h_{Q}$ uniformly on $\partial A_{r_{i}}$. Therefore, from the maximum principle, $h_{P}$ converges to $h_{Q}$ uniformly on $\{|z|=1\}=f\left(C_{i}\right)$ and we have

$$
\lim _{P \rightarrow Q} \int_{|z|=1} \varphi \circ f^{-1} \theta_{P}=\int_{|z|=1} \varphi \circ f^{-1} \theta_{Q} .
$$

Noting that

$$
\int_{C_{i}} \varphi \omega_{\hat{P}_{0}, P}=\int_{|z|=1} \frac{\varphi \circ f^{-1}(z)}{z-f(P)} d z+\int_{|z|=1} \varphi \circ f^{-1} \theta_{P},
$$

we verify that the behavior of $F(P)$ as $P \rightarrow Q \in C_{i}$ is determined by that of the Cauchy integral of $\varphi \circ f^{-1}$ on the unit circle. This implies the conclusion of $(2)$.

Proof of (3). The proof of the non-tangential limits is done by the localization as in (2) since the statement is true when $R$ is the unit disk (cf. [4]).

Now, we show the second statement of (3) holds. Since $\varphi \in L^{p}(\partial R)$ $(p>1)$, from Proposition 2 we have

$$
\varphi=f_{1}+\overline{f_{2}}+m
$$

on $\partial R$, where $f_{1} \in H^{p}(R), f_{2} \in H_{0}^{p}(R)$ and $m \in M\left(\delta^{-1}\right)$. (If $p=\infty$, we may take any finite number greater than 1 as $p$ in (7.4).) Then, from Cauchy's integral formula for $H^{p}(R)$, we have

$$
\frac{1}{2 \pi i} \int_{\partial R} f_{1} \omega_{\hat{P}_{0}, P}=f_{1}(P)
$$

for $P \in R$. Since $F_{2}:=\overline{f_{2} \circ \pi}$ is in $H^{p}(\hat{R} \backslash \bar{R})$ and $F_{2}=\bar{f}_{2}$ on $\partial R$, we have

$$
\frac{1}{2 \pi i} \int_{\partial R} \overline{f_{2}} \omega_{\hat{P}_{0}, P}=-F_{2}\left(\hat{P}_{0}\right)=-\overline{f_{2}\left(P_{0}\right)}=0 .
$$

Let $Q_{1}^{n_{1}} \ldots Q_{k}^{n_{k}}$ be the polar divisor of $m$ in $R$ and

$$
m\left(z_{j}\right)=\sum_{l=1}^{n_{j}} \frac{d_{j, l}}{z^{l}}+\sum_{l=0}^{\infty} d_{j, l}^{+} z^{l} \quad(j=1,2, \ldots, k)
$$

the Laurent expansion of $m$ at $Q_{j}$ with respect to a local coordinate $z_{j}$ of $Q_{j}$ with $z_{j}\left(Q_{j}\right)=0$. For $P \neq Q_{j}$, we put

$$
\omega_{\hat{P}_{0}, P}=\sum_{l=0}^{\infty} a_{j, l}(P) z_{j}^{l} d z_{j}
$$


near $Q_{j}$. Then, we obtain

$$
\frac{1}{2 \pi i} \int_{\partial R} m \omega_{\hat{P}_{0}, P}=m(P)+\sum_{j=1}^{k} \sum_{l=1}^{n_{j}} d_{j, l} a_{j-1, l}(P),
$$

if $P \neq Q_{j}(j=1,2, \ldots, k)$. Therefore, we obtain

$$
F(P)=f_{1}(P)+m(P)+\sum_{j=1}^{k} \sum_{l=1}^{n_{j}} d_{j, l} a_{j-1, l}(P),
$$

if $P \in R \backslash \bigcup_{j=1}^{k} Q_{j}$. Hence, it follows from Lemma 1 that the non-tangential limit $F_{+}(Q)$ of $F$ from $R$ exists for almost all $Q$ on $\partial R$. By the same argument, $F_{-}$exists almost everywhere on $\partial R$.

On the other hand, for $P \in R$ and $P^{\prime} \in \pi(R)$,

$$
F(P)-F\left(P^{\prime}\right)=f_{1}(P)+\overline{f_{2}\left(\pi\left(P^{\prime}\right)\right)}+\frac{1}{2 \pi i} \int_{\partial R} m \omega_{P, P^{\prime}},
$$

because $\omega_{P^{\prime}, P}=\omega_{\hat{P}_{0}, P}-\omega_{\hat{P}_{0}, P}$. By the same argument as in (7.5), we obtain

$$
\frac{1}{2 \pi i} \int_{\partial R} m \omega_{P^{\prime}, P}=m(P)+\sum_{j=1}^{k} \sum_{l=1}^{n_{j}} d_{j, l} b_{j-1, l}\left(P, P^{\prime}\right),
$$

where $\omega_{P^{\prime}, P}=\sum_{l=0}^{\infty} b_{j, l}\left(P, P^{\prime}\right) z_{j}^{l} d z_{j}$ at $Q_{j}(j=1,2, \ldots, k)$. Since $b_{j, l}\left(P, P^{\prime}\right) \rightarrow 0$ as $P, P^{\prime} \rightarrow Q$, we have

$$
\lim _{P, P^{\prime} \rightarrow Q} \frac{1}{2 \pi i} \int_{\partial R} m \omega_{P^{\prime}, P}=m(Q) .
$$

Therefore, from (7.6), we verify

$$
F_{+}-F_{-}=\varphi
$$

almost everywhere on $\partial R$ and the proof is completed.

\section{REFERENCES}

[1] Aikawa, H., Modulus of continuity of the Dirichlet solutions, Bull. London Math. Soc. 42 (2010), 857-867.

[2] Bikčantaev, I. A., Analogues of a Cauchy kernel on a Riemann surface and some applications of them, Mat. Sb. (N.S.) 112 (154), no. 2 (6) (1980), 256-282 (Russian); translation in Math. USSR Sb. 40, no. 2 (1981), 241-265.

[3] Block, I. E., The Plemelj theory for the class $\Lambda^{*}$ of functions, Duke Math. J. 19 (1952), 367-378.

[4] Duren, P. L., Theory of $H^{p}$ Spaces, Academic Press, New York-San FranciscoLondon, 1970.

[5] Farkas, H. M., Kra, I., Riemann Surfaces, Springer-Verlag, New York-HeidelbergBerlin, 1980.

[6] Gakhov, F. D., Boundary Value Problems, Pergamon Press, Oxford-New York-Paris, 1966. 
[7] Garnett, J. B., Bounded Analytic Functions, Academic Press, New York-London, 1981.

[8] Gong, S., Integrals of Cauchy type on the ball, International Press, Cambridge, 1993.

[9] Guseinov, E. G., The Plemelj-Privalov theorem for generalized Hölder classes, Mat. Sb. 183, no. 2 (1992), 21-37 (Russian); translation in Russian Acad. Sci. Sb. Math. 75 (1993), 165-182.

[10] Heins, M., Hardy Classes on Riemann Surfaces, Springer-Verlag, Berlin-New York, 1969.

[11] Shiga, H., Riemann mappings of invariant components of Kleinian groups, J. London Math. Soc. 80 (2009), 716-728.

[12] Shiga, H., Modulus of continuity, a Hardy-Littlewood theorem and its application, RIMS Kokyuroku Bessatsu, 2010, 127-133.

[13] Pommerenke, C., Boundary Behaviour of Conformal Maps, Springer-Verlag, Berlin, 1992.

[14] Walsh, J. L., Polynomial expansions of functions defined by Cauchy's integral, J. Math. Pures Appl. 31 (1952), 221-244.

Hiroshige Shiga

Department of Mathematics

Tokyo Institute of Technology

e-mail: shiga@math.titech.ac.jp

Received September 18, 2011 